\title{
Design and test of a novel accelerometer made-up of an optical-fiber embedded within a polymer resin
}

\author{
Pierre Tihon $^{1, \mathrm{a}}$, Olivier Verlinden ${ }^{1, \mathrm{~b}}$, Georges Kouroussis ${ }^{1, \mathrm{c}}$, Patrice Mégret $^{2, \mathrm{~d}}$ and Marc Wuilpart ${ }^{2, \mathrm{e}}$ \\ 1 University of Mons, Department of Theoretical Mechanics, Dynamics and Vibrations, 31 Boulevard Dolez, \\ 7000 Mons, Belgium \\ 2 University of Mons, Department of Electromagnetism and Telecommunication, 31 Boulevard Dolez, 7000 Mons, \\ Belgium
}

\begin{abstract}
This paper presents a transducer for an optical-fiber accelerometer based on a polarization analysis. The transducer is made up of a fiber section embedded within a resin placed between two metallic pieces. Due to the acceleration, the resin is crushed between the metallic pieces, deforming the fiber section and inducing birefringence in the latter. This birefringence modifies the light polarization state, which can be used as an acceleration measurement. The sensor characteristics (sensitivity and resonance frequency) are numerically and experimentally determined. Sine accelerations at $120 \mathrm{~Hz}$ with amplitudes going from $5 \mathrm{~m} / \mathrm{s}^{2}$ to $13 \mathrm{~m} / \mathrm{s}^{2}$ have been successfully measured. The resonance frequency for the transducer crushing mode is above $5000 \mathrm{~Hz}$, but low-frequency vibration modes exist, disturbing the measurements.
\end{abstract}

\section{Introduction}

Vibration measurements are widely used in the fields of civil or mechanical engineering, for example for maintenance purposes. While the piezo-electric and the piezo-resistive accelerometers are widely used, different studies [1-5] have considered the use of optical fibers to measure dynamic accelerations. Optical fibers allow distributed and quasi-distributed measurements. They are furthermore immune to humidity or electromagnetic perturbations. Among the different light properties that can be used for measurement applications (such as power, wavelength, polarization, phase), the use of polarization usually leads to simple measurement set-ups, especially compared to the interferometry techniques, where a very narrow light source is needed.

The principle of acceleration measurements with the use of polarization will be detailed in Sect. 2. It is based on the deformation of an optical-fiber with a mechanical transducer submitted to the vibration. We presented in $[4,5]$ acceleration measurements based on a transducer that consisted of an aluminium base placed on the vibrating structure. A flexible beam is fixed at one extremity to the base. The second extremity crushes the fiber against the base. However, this transducer was heavy (mass of 80 grams) and cumbersome. In this paper, we propose a new smaller transducer, where the fiber is embedded within a polymer resin that supports a seismic mass. The aluminium and resin sensor are presented in

\footnotetext{
a e-mail: pierre.tihon@umons .ac . be

b e-mail: olivier.verlinden@umons.ac.be

c e-mail: georges.kouroussis@umons .ac . be

d e-mail: patrice.megret@umons.ac.be

e e-mail: marc.wuilpart@umons.ac . be
}

Fig. 1. Simulations and experimental results are presented to study the usability of the resin transducer. The sensor sensitivity and resonance frequency will be determined.

In this paper, monomode optical fibers are used. These fibers consist of a core in doped silica, with a diameter of $8 \mu \mathrm{m}$, surrounded by a layer of silica (with a different doping), with a diameter of $125 \mu \mathrm{m}$. This last silica layer is surrounded by a protective layer in polymer with a diameter of $250 \mu \mathrm{m}$. Most of the light power propagates only in the fiber core of $8 \mu \mathrm{m}$.

\section{Theoretical background}

Light is composed by an electric field $\mathbf{E}$ and a magnetic field $\mathbf{H}$, perpendicular to each other and to the direction of propagation. The state of polarization (SOP) characterizes the evolution of the electric field vector position along the direction of propagation. In the most general case, the extremity of the electric vector $\mathbf{E}$ follows an elliptical path, the polarization is called elliptical. If the ellipse is flattened and becomes a line segment, the polarization is linear.

In a perfectly symmetric fiber, all the states of polarization propagate with the same speed. However, if the symmetry is broken, for example if the fiber is deformed, the different SOPs will have different speeds. The fastest and the lowest SOPs are the eigenmodes. Let us denote $n_{x}$ and $n_{y}$ the refractive indices corresponding to the fastest and lowest modes respectively. The birefringence $\beta$ is defined as

$$
\beta=\frac{2 \pi}{\lambda}\left(n_{x}-n_{y}\right)
$$

where $\lambda$ is the light wavelength. The birefringence corresponds to the variation of the optical phase per 


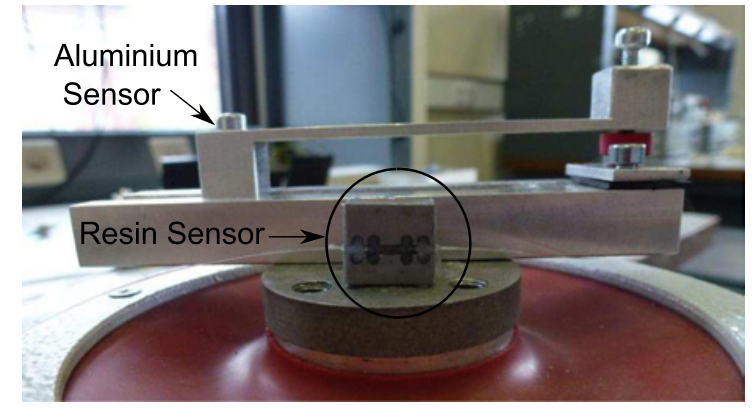

Figure 1. Aluminium and resin sensors.

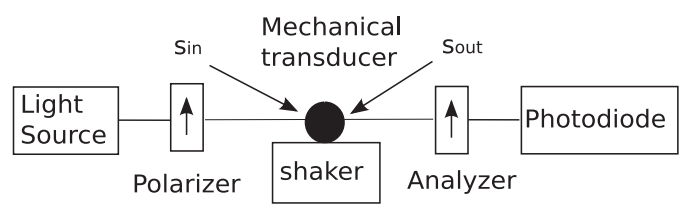

Figure 2. Experimental set-up.

unit of length that occurs between the fast and slow eigenmodes propagating through the fiber. If the fiber has a length $L_{f}$, the optical phase delay $\delta$ that appears between the eigenmodes will be $\delta=\beta \times L_{f}$. The effect of the birefringence is to modify the state of polarization. If polarized light is sent into an optical fiber submitted to a birefringence variation, the output SOP varies with the birefringence.

An optical fiber has an intrinsic birefringence $\beta_{0}$. This birefringence is modified when the fiber is submitted to deformation. It can be shown that if the fiber is crushed, linear birefringence occurs (i.e. birefringence with linear eigenmodes). In this case, the link between the stresses that occur in the fiber center (where the light propagates) and the stress-induced birefringence variation $\Delta \beta$ is given by [6]:

$$
\Delta \beta=-\frac{\pi n^{3}}{\lambda}\left(p_{11}-p_{12}\right) \frac{1+v}{E}\left(\sigma_{x}-\sigma_{y}\right)
$$

where $n$ is the mean refractive index, $p_{11}$ and $p_{12}$ the photo-elastic coefficients, $E$ the Young's modulus and $v$ the Poisson's ratio of the fiber material. For silica, we have $n=1.45, p_{11}=0.121, p_{12}=0.27, E=70 \mathrm{GPa}$ and $v=0.16 . \sigma_{x}$ and $\sigma_{y}$ are the stresses that occur in the fiber center, in the directions parallel and perpendicular to the applied force.

It is then possible to determine an acceleration through polarimetric measurements with the set-up of Fig. 2. A laser source sends polarized light into an optical fiber. The SOP can be set with a polarizer. At some point, the fiber is deformed by a mechanical transducer submitted to the vibration. This deformation induces stress-induced birefringence variation $\Delta \beta$ within the fiber, that modifies the state of polarization. A second polarizer, called analyzer in the figure, is placed after the transducer. Due to the SOP modification, the output power varies, which can be used as an acceleration measurement $[4,5]$.

With this set-up however, the sensor sensitivity depends on the SOP $\mathbf{s}_{i n}$ at the transducer input. For example, if the SOP is one of the eigenmodes of the

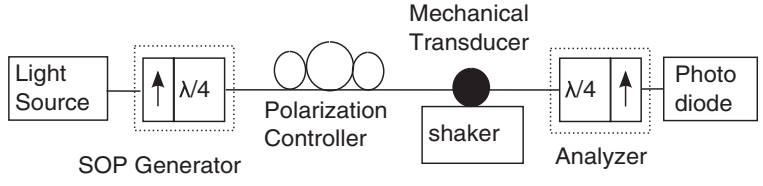

Figure 3. Experimental set-up.

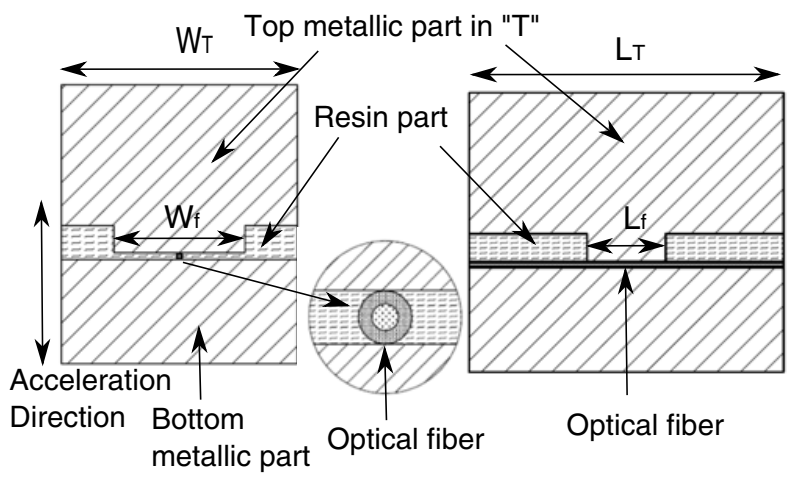

Figure 4. Resin sensor. Left: front view. Right: side view.

stress-induced birefringence, the sensitivity is zero. It is very difficult to impose this SOP because the fiber intrinsic birefringence is unknown and can vary with time. To be able to measure accelerations with a constant sensitivity, it is necessary to combine different optical measurements. We described in $[7,8]$ a technique that allows the measurement of one acceleration through twelve polarimetric measurements. The technique uses the set-up of Fig. 3. Three different SOPs are successively sent into the fiber, thanks to an SOP generator that consists of a rotating polarizer followed by a quarter-wave plate. For each of the input SOPs, the analyzer (that consists here of a quarter-wave plate followed by a rotating polarizer) is set on four different configurations and the light output power is measured. The combination of the 12 power measurements gives a measurement of the optical phase $\delta=\beta \times L_{f}$ that does not depend on the fiber intrinsic birefringence $[7,8]$. As the variation of $\delta$, denoted $\Delta \delta$, is proportional to the acceleration, the latter can be measured. This technique will be used in this paper.

\section{Transducer description}

The designed resin sensor is presented in Fig. 4. A front view is shown on the left, a side view on the right. The fiber is placed between two metallic pieces. The piece at the bottom is fixed to the vibrating structure. The piece at the top is used as a seismic mass. Both pieces are hold together with a resin in which the fiber is embedded. The stresses due to the vibration of the seismic mass are spread within the resin and the fiber. In order to focus more efficiently the stresses into the fiber, the top piece has the form of a " $T$ ". The horizontal part of the " $T$ " is the seismic mass itself while the vertical part is in direct contact with the fiber. In the figure, the width of this vertical part is called $W_{f}$ and its length is called $L_{f}$. $L_{f}$ corresponds to the length of the crushed fiber. The sensor total length is denoted $L_{T}$ and its width $W_{T}$. In the figure, the measured acceleration direction is vertical. 
Table 1. Transducers characteristics.

\begin{tabular}{cccccc}
\hline Material & $L_{T}(\mathrm{~mm})$ & $W_{T}(\mathrm{~mm})$ & $L_{f}(\mathrm{~mm})$ & $W_{f}(\mathrm{~mm})$ & Seismic mass $(\mathrm{g})$ \\
\hline Polyester & 12 & 9 & 2 & 1.2 & 3.5 \\
Silicone & 12 & 9 & 3 & 5 & 4 \\
Silicone & 11 & 8 & 2.85 & 1.9 & 4 \\
\hline
\end{tabular}

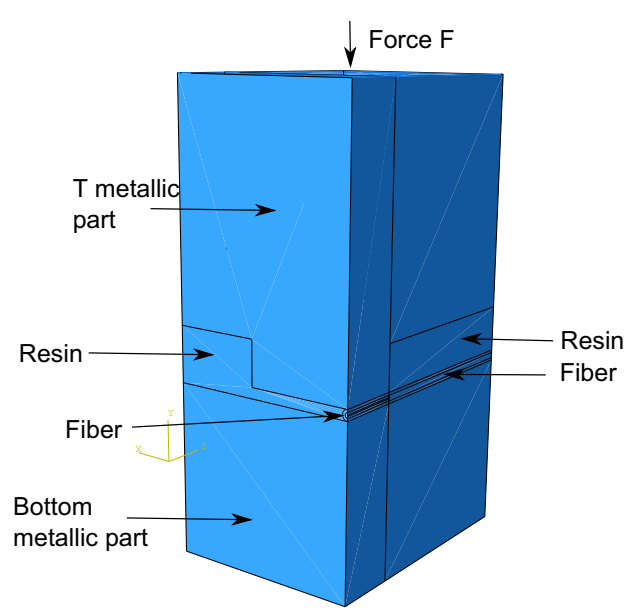

Figure 5. Abaqus model.

The first transducer was built with a polyester resin. However, as the polyester is rather stiff (Young's modulus of $160 \mathrm{MPa}$ ), the sensor sensitivity was low. The next transducers have been built with a silicone resin, which is much smoother (Young's modulus of 3.5 MPa). A single polyester transducer and two silicone transducers have been built. Their characteristics are presented in Table 1 . The first silicone sensor has a larger " $T$ " than the second one (the widths $W_{f}$ are respectively equal to $5 \mathrm{~mm}$ and $1.9 \mathrm{~mm})$. The large width has been chosen in order to ease the positioning of the fiber under the " $T$ ". The thin width has been chosen in order to concentrate the stresses into the fiber. It was then necessary to be accurate in the transducer fabrication, to avoid that the fiber be outside the "T". Those two transducers will be later denoted "transducer with large crushing part" and "transducer with thin crushing part" respectively. All the resin transducers have an approximate weight of 10 grams.

\section{Simulation results}

The resin sensors have been modeled with the Abaqus software, in order to compute their sensitivities and resonance frequencies.

\subsection{Sensitivity}

The sensor model is presented in Fig. 5. Only a quarter of the transducer is modeled, taking into account the symmetry conditions, to reduce the computation time. The top metallic part is statically crushed by a force $F$ of $1.25 \mathrm{~N}$, that represents the force induced by the seismic mass motion. The stresses $\sigma_{x}$ and $\sigma_{y}$ in the fiber core are computed, giving the birefringence $\beta$ through Eq. (2).

The acceleration $A$ corresponding to the crushing force $F$ depends on the considered seismic mass $m$ through formula: $A=\frac{F}{m}$. The sensor sensitivity is defined as the optical phase shift variation $\Delta \delta$ divided by the acceleration amplitude $A$. Denoting $L_{f}$ the length of the crushed fiber section, we have:

$$
\text { Sensitivity }=\frac{\Delta \delta}{A}=\frac{\Delta \beta L_{f}}{A}
$$

The results are shown in Tables 2 to 4 for the polyester and silicone transducers. The computed sensitivities vary from $6.59 \times 10^{-5} \mathrm{rad} /\left(\mathrm{m} / \mathrm{s}^{2}\right)$ to $3.8 \times 10^{-4} \mathrm{rad} /\left(\mathrm{m} / \mathrm{s}^{2}\right)$. The use of silicone instead of polyester increases the sensitivity, due to the lower stiffness of silicone (Young's modulus of 3.5 MPa instead of $160 \mathrm{MPa}$ ). Furthermore, reducing the width of the crushing part has a positive influence too, as the stresses are more focused.

\subsection{Resonance frequencies}

The main vibration modes computed with Abaqus for the silicone transducers are presented in Figs. 6 to 9, where the whole transducer has been modeled. We see that the top metallic piece can have different kinds of rigid body motion: torsion, bending or crushing. The corresponding resonance frequencies are presented in Tables 5 and 6 for the sensors with large and thin crushing parts respectively. The resonance frequencies for the crushing modes, where the seismic mass vibrates in the direction of the acceleration, reach $5410 \mathrm{~Hz}$ and $5780 \mathrm{~Hz}$. The resonance frequencies for the other modes (torsion, bending) are lower, between 820 and $1750 \mathrm{~Hz}$.

\section{Measurement results}

\subsection{Resonance frequencies}

This section presents the experimental results concerning the frequency response of the silicone transducers. Sine sweep accelerations are applied to the silicone transducers, with the large or the thin crushing part. The applied frequency bandwidth goes from $0 \mathrm{~Hz}$ to $7500 \mathrm{~Hz}$. A single SOP is sent into the fiber. The output power variation amplitude is measured at each frequency, and compared with the applied acceleration measured with a reference piezo-electric accelerometer.

The experimental results are presented in Figs. 10 and 11 . In the figures, the frequency response function (the FRF, defined as the amplitude of the sensor output divided by the amplitude of the applied acceleration for each frequency) is shown. The dotted lines present the amplitude of the FRFs. The solid lines present the phase of the FRFs (the phase-shift between the sensor output and the applied acceleration). A resonance is associated to a peak in the amplitude and to a phase variation. The main resonances are shown with arrows. 
Table 2. Simulated sensitivity of the polyester transducer.

\begin{tabular}{cccccc}
\hline Force $(\mathrm{N})$ & $\Delta \sigma\left(\mathrm{N} / \mathrm{m}^{2}\right)$ & $\beta(\mathrm{rad} / \mathrm{m})$ & Seismic mass $(\mathrm{g})$ & $\mathrm{A}\left(\mathrm{m} / \mathrm{s}^{2}\right)$ & Sensitivity $\left(\mathrm{rad} /\left(\mathrm{m} / \mathrm{s}^{2}\right)\right)$ \\
\hline 1.25 & $-7.4 \times 10^{5}$ & $1.2 \times 10^{1}$ & 3.5 & 357.14 & $6.59 \times 10^{-5}$ \\
\hline
\end{tabular}

Table 3. Simulated sensitivity of the silicone transducer - large crushing part.

\begin{tabular}{cccccc}
\hline Force $(\mathrm{N})$ & $\Delta \sigma\left(\mathrm{N} / \mathrm{m}^{2}\right)$ & $\beta(\mathrm{rad} / \mathrm{m})$ & Seismic mass $(\mathrm{g})$ & $\mathrm{A}\left(\mathrm{m} / \mathrm{s}^{2}\right)$ & Sensitivity $\left(\mathrm{rad} /\left(\mathrm{m} / \mathrm{s}^{2}\right)\right)$ \\
\hline 1.25 & $1.99 \times 10^{6}$ & 31.6 & 4 & 312.5 & $3.03 \times 10^{-4}$ \\
\hline
\end{tabular}

Table 4. Simulated sensitivity of the silicone sensor - thin crushing part.

\begin{tabular}{cccccc}
\hline Force $(\mathrm{N})$ & $\Delta \sigma\left(\mathrm{N} / \mathrm{m}^{2}\right)$ & $\beta(\mathrm{rad} / \mathrm{m})$ & Seismic mass $(\mathrm{g})$ & $\mathrm{A}\left(\mathrm{m} / \mathrm{s}^{2}\right)$ & Sensitivity $\left(\mathrm{rad} /\left(\mathrm{m} / \mathrm{s}^{2}\right)\right)$ \\
\hline 1.25 & $2.34 \times 10^{6}$ & 37 & 4 & 277.78 & $3.8 \times 10^{-4}$ \\
\hline
\end{tabular}

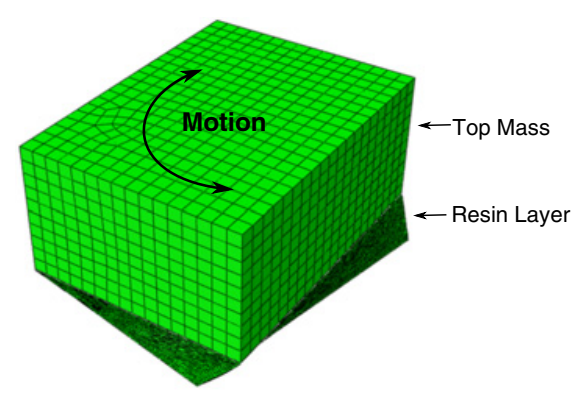

Figure 6. Torsion mode.

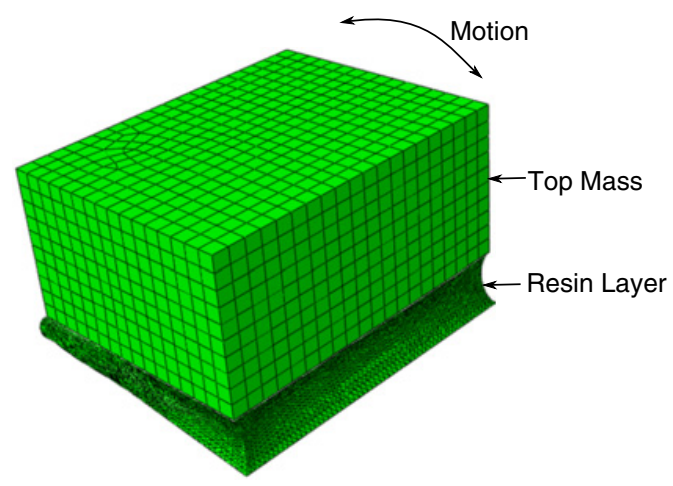

Figure 7. First bending mode.

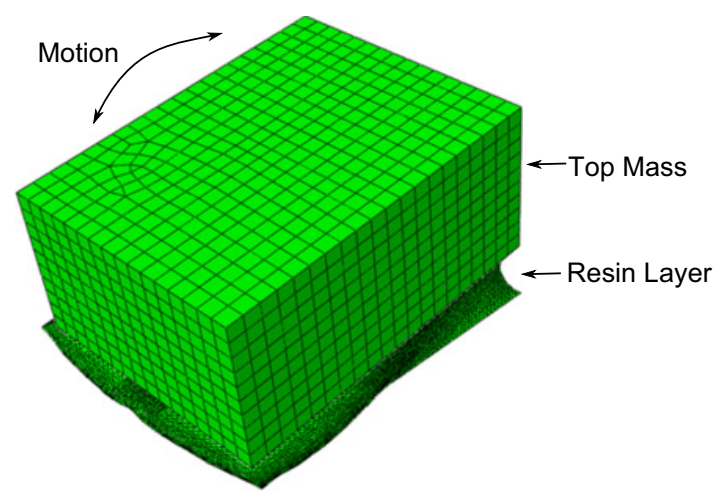

Figure 8. Second bending mode.

As we can see, there are always different resonance frequencies for each measurement. On each curve, the frequency corresponding to the first crushing mode is clearly visible, as it is the mode corresponding to the acceleration direction. The resonance frequencies in crushing reach $3950 \mathrm{~Hz}$ and $6025 \mathrm{~Hz}$. Tables 7

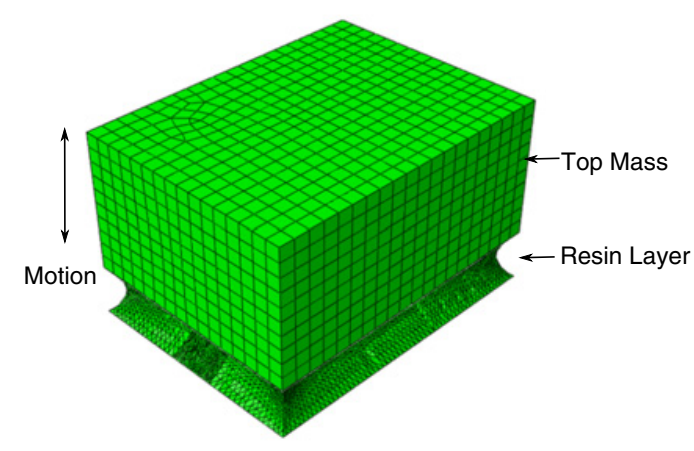

Figure 9. Crushing mode.

Table 5. Simulated resonance frequencies for the large crushing sensor.

\begin{tabular}{ccccc}
\hline & Mode 1 & Mode 2 & Mode 3 & Mode 4 \\
\hline Type of motion & Torsion & Bending & Bending & Crushing \\
Frequency $(\mathrm{Hz})$ & 918 & 1118 & 1750 & 5780 \\
\hline
\end{tabular}

Table 6. Simulated resonance frequencies for the thin crushing sensor.

\begin{tabular}{ccccc}
\hline & Mode 1 & Mode 2 & Mode 3 & Mode 4 \\
\hline Type of motion & Torsion & Bending & Bending & Crushing \\
Frequency $(\mathrm{Hz})$ & 870 & 997 & 1690 & 5410 \\
\hline
\end{tabular}

and 8 compare the experimental and simulated frequencies for the large and thin crushing part silicone sensors respectively.

Ideally the crushing mode would be the only one present. However, several peaks are present at lower frequencies and correspond to bending or torsion modes of the seismic mass. They can be excited if the seismic mass is not perfectly centered on the sensor, if the resin is not homogeneous or simply if the excitation is not exactly oriented along the sensor height (for example if the glue between the sensor and the vibrating shaker is not perfectly plane).

Figure 12 presents the amplitude of the FRF for an applied sweep sine that has a maximal frequency of $200 \mathrm{~Hz}$, in order to have a zoom on the sensor behaviour at low frequencies. The sensor is in silicone with the thin crushing. The measurement has been repeated three times, and the obtained curves are very jagged. It seems that several low frequency modes exist, in addition to the main 


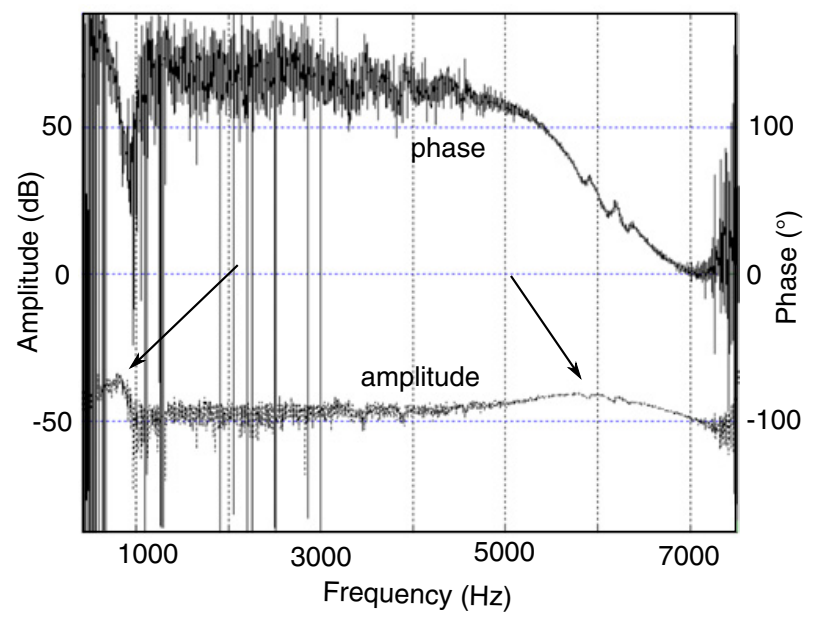

Figure 10. FRF for the silicone sensor with large crushing.

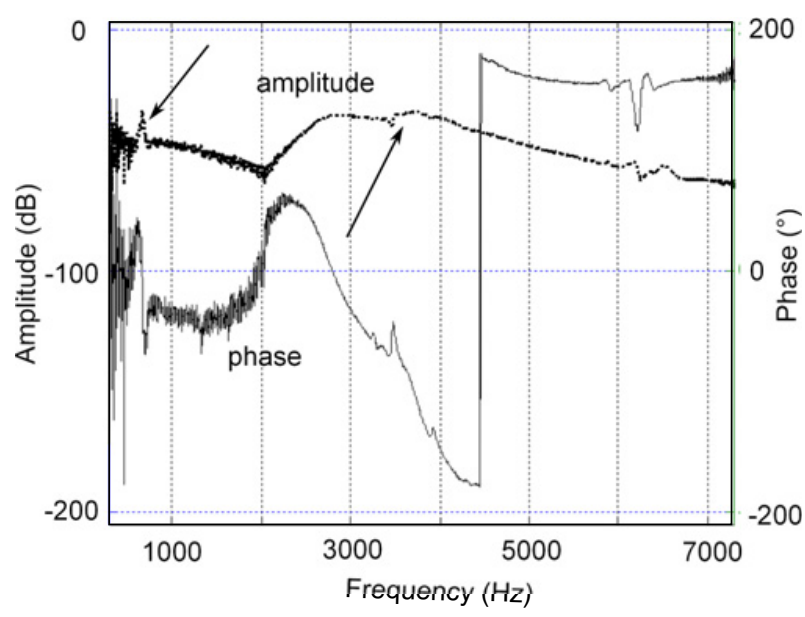

Figure 11. FRF for the silicone sensor with thin crushing.

Table 7. Comparison between the measured and simulated frequencies for the large crushing sensor.

\begin{tabular}{lcccc}
\hline & Mode 1 & Mode 2 & Mode 3 & Mode 4 \\
\hline Type of motion & Torsion & Bending & Bending & Crushing \\
Simulated Frequency & 918 & 1118 & 1750 & 5780 \\
Measured Frequency & 820 & - & - & 6025 \\
\hline
\end{tabular}

Table 8. Comparison between the measured and simulated frequencies for the thin crushing sensor.

\begin{tabular}{lcccc}
\hline & Mode 1 & Mode 2 & Mode 3 & Mode 4 \\
\hline Type of motion & Torsion & Bending & Bending & Crushing \\
$\begin{array}{l}\text { Simulated Frequency } \\
\text { (Hz) }\end{array}$ & 870 & 997 & 1690 & 5410 \\
$\begin{array}{l}\text { Measured Frequency } \\
(\mathrm{Hz})\end{array}$ & 662 & - & - & 3950 \\
\hline
\end{tabular}

bending and crushing modes presented in Sect. 4.2. This behaviour should be avoided as it means that the sensor sensitivity greatly depends on the frequency. For example, the sensitivity at $188 \mathrm{~Hz}$ (where a resonance peak exists) is three times higher than the one at $120 \mathrm{~Hz}$.

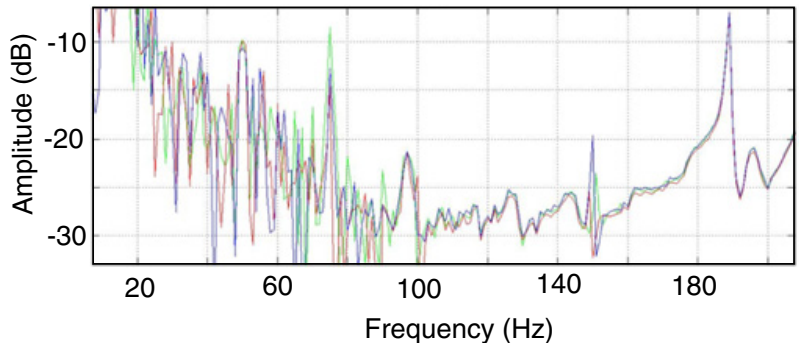

Figure 12. FRF for the silicone sensor with thin crushing (Zoom).

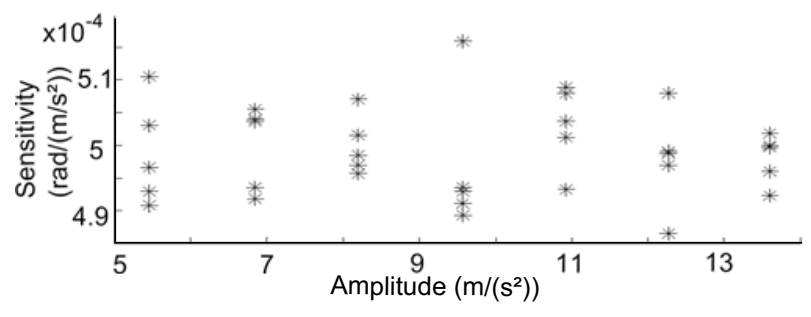

Figure 13. Sensitivity - Silicone sensor with thin crushing.

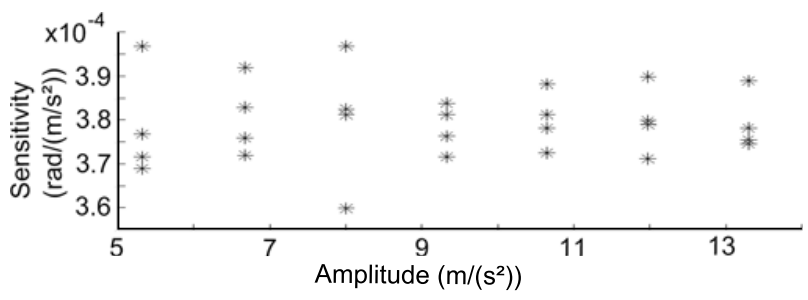

Figure 14. Sensitivity - Silicone sensor with thin crushing.

Table 9. Measured and simulated sensitivities.

\begin{tabular}{ccc}
\hline & $\begin{array}{c}\text { Measured sensitivity } \\
\left(\mathrm{rad} /\left(\mathrm{m} / \mathrm{s}^{2}\right)\right)\end{array}$ & $\begin{array}{c}\text { Simulated sensitivity } \\
\left(\mathrm{rad} /\left(\mathrm{m} / \mathrm{s}^{2}\right)\right)\end{array}$ \\
\hline $\begin{array}{c}\text { Silicone } \\
\text { large crushing } \\
\text { Silicone } \\
\text { thin crushing }\end{array}$ & $5 \times 10^{-4}$ & $3.03 \times 10^{-4}$ \\
\hline
\end{tabular}

\subsection{Sensitivities}

The polyester and silicone sensors sensitivities have been measured using the experimental set-up of Fig. 3. Sine accelerations at $120 \mathrm{~Hz}$ are applied and 12 polarimetric measurements are used for one acceleration measurement, as explained in Sect. 2. The results obtained with the polyester transducer were too noisy, and only the results for the silicone sensors are presented. Seven different acceleration levels are applied, going from 5 to $13 \mathrm{~m} / \mathrm{s}^{2}$. After each series of seven acceleration measurements, the set of three input SOPs sent into the transducer is modified (with the polarization controller of Fig. 3), and new measurements are performed. This is done for four (in Fig. 14) or five (in Fig. 13) different sets of input SOPs. The twelve polarimetric measurements give the stressinduced optical delay variation $\Delta \delta$ [7]. This variation is proportional to the applied acceleration $[4,5]$.

The results are shown in Figs. 13 and 14 which present for each acceleration amplitude the measured sensitivity obtained with the four or five measurements. The 
sensitivity is defined as the amplitude of the optical delay $\delta$ divided by the amplitude of the applied acceleration, as defined in Eq. (3). The results are summarized in Table 9 where the experimental and simulated results are compared. Even if the orders of magnitude of the sensitivities are the same in the experiments as in the simulations, differences exist. Contrary to the simulations, the silicone sensor with a large crushing part has a higher sensitivity than the one with the thin crushing part $(5 \times$ $10^{-4} \mathrm{~m} / \mathrm{s}^{2}$ instead of $3.88 \times 10^{-4} \mathrm{~m} / \mathrm{s}^{2}$ ). The differences can come from the facts that the silicone properties are not exactly the same from one transducer to another, that the fiber may be more or less well centered under the "T" metallic piece, and that the fiber may be damaged during the sensor fabrication.

\subsection{Conclusion}

This paper presents a novel transducer for acceleration measurements. The transducer consists of an optical fiber embedded within a resin that supports a seismic mass. The transducer characteristics (sensitivity, resonance frequency) are numerically and experimentally studied. The numerical and experimental results are rather similar, even if differences exist, due to uncertainties on the resin mechanical properties and on the sensor fabrication. It is shown that a smooth resin (such as silicone) has to be used in order to increase the sensitivity. Minimal accelerations of $5 \mathrm{~m} / \mathrm{s}^{2}$ have been measured. The crushing mode resonance frequency is quite high (above $5000 \mathrm{~Hz}$ ), however smaller resonances exist, due to the presence of bending modes within the sensor. Efforts have still to be done to diminish the influence of the low-frequency bending modes, in order to obtain a sensor sensitivity that does not depend on the acceleration frequencies. Future work will deal with the design of a new transducer that has an external shell in order to increase the bending modes resonance frequencies. Measurements with several transducers put on the same fiber will also be performed to test the feasibility of distributed measurements.

\section{References}

[1] F. Qin, H. Li, W. Fan and Q. Sheng, Chin. Opt. Lett. 7, 556-559 (2009)

[2] Z. Zhang and X. Bao, Optics Express 16, 1024010247 (2008)

[3] J.-G. Liu, C. Schmidt-Hattenberger, G. Borm, Measurement 32, 151-161 (2002)

[4] P. Tihon, N. Linze, O. Verlinden and M. Wuilpart, International Conference on Sound and Vibration, 607-614 (2011)

[5] N. Linze, P. Tihon, O. Verlinden, P. Mégret and M. Wuilpart, Applied Optics 51, 6997-7004 (2012)

[6] R. Ulrich, S.C. Rashleigh and W. Eickhoff, Optics Letters 5, 273-275 (1980)

[7] P. Tihon, N. Linze, O. Verlinden, P. Mégret and M. Wuilpart, AIP Conference Proceedings 1569, 471 (2013)

[8] P. Tihon, O. Verlinden, P. Mégret and M. Wuilpart, Applied Optics 53, 5029-5036 (2014) 